\title{
Mobility in the built environment: Age-related changes in gait characteristics when walking on complex terrain
}

\author{
Ian McCarthy ${ }^{1 *}$, Tatsuto Suzuki $^{1}$, Nick Tyler ${ }^{1}$, Catherine Holloway ${ }^{2}$ \\ 1 Pedestrian Accessibility \& Movement Environment Laboratory (PAMELA), Department of Civil, Environmental and Geomatic \\ Engineering, University College London, London, UK 2 UCL Interaction Centre, Department of Computer Science, University College \\ London, London, UK
}

\begin{abstract}
Background: An understanding of how common features in the built environment influence how people walk is needed to maintain mobility for older people.

Methods: The study included 71 healthy subjects with an age range of 18 to 92 years. Using inertial measurement units, participants' gaits were assessed while walking across a complex terrain created in a controlled laboratory environment.

Results: Participants found stair climbing and stepping on obstacles to be the most challenging activities, as judged by step time. These activities also showed the most significant age-related changes, with significant effects in both step time and shank angle at touch down being observed from around the age of 60 years.
\end{abstract}

Conclusions: The changes observed in this study are consistent with decreasing muscle power causing limited ability to negotiate stairs.

Citation: McCarthy I, Suzuki T, Tyler N, Holloway C (2016) Mobility in the built environment: Age-related changes in gait characteristics when walking on complex terrain. Healthy Aging Research 5:10.

Received: December 22, 2015; Accepted: May 2, 2016; Published: August 1, 2016

Copyright: (C) 2016 McCarthy et al. This is an open access article distributed under the terms of the Creative Commons Attribution License, which permits unrestricted use, distribution, and reproduction in any medium, provided the original work is properly cited.

Competing interests: The authors have declared that no competing interests exist.

Sources of funding: The study was funded through general support for activities within PAMELA.

*Email: i.mccarthy@ucl.ac.uk

\section{Introduction}

The proportion of older people in both developed and developing world populations is increasing significantly. Understanding how mobility changes with age can help older people to maintain accessibility and independence for as long as possible. Several studies have investigated the walking ability of elderly persons; however, these are usually performed in gait laboratories, whereby people walk at a constant velocity over a smooth, flat surface. Several studies have also linked urban infrastructure design to accessibility; however, these tend to examine accessibility in a macro setting, investigating the number and types of journeys. Journeys are completed by successfully navigating and ambulating in the built and natural environment; this requires walking over uneven surfaces, dealing with steps and gradients, and frequent adjustments to gait pattern are required.

Several studies have investigated more complex environments or activities, such as stepping accuracy [1], crossing obstacles [2, 3], stair climbing [4], inclined walkways [5], and multi-surface terrains [6]. Such studies have emphasised the need to further understand the problems of motor control associated with such environments.

To maintain a healthy lifestyle, and for older people to maintain independence for as long as possible, the 
built environment should attempt to encourage physical activity [7]. The degree to which individuals utilise opportunities for activity depends on their capabilities. In the capabilities model, a person's objective is to undertake the activities they want to do, and their ability to achieve this depends on the relationship between the capabilities required by the activity and its associated environment(s), and the individual's capabilities [8]. Thus, accessibility is a function of the interactions between required and provided capabilities, and this brings to the fore the need to measure and evaluate these capabilities. After evaluation, it will then be possible to identify any areas of deficit that need to be addressed. There are two approaches to deal with this: i) to modify the capabilities provided by the individual (e.g. total joint replacement to reduce pain associated with osteoarthritis) so that people can better address the challenges of the environment; or ii) to modify the capabilities required by the activity (e.g. by providing step-free access to local transport facilities) so that the required capabilities are reduced.

Individual functions decline with age and disease, limiting the ability to participate in desired activities. It is therefore important to understand age-related changes in functional abilities. It is also useful to separate the effects of ageing per se (primary ageing effects) from those of chronic diseases (e.g. diabetes, cardiopulmonary disease, osteoarthritis) that are much more prevalent in the elderly, so that rehabilitation is better directed to improving function. In a study of walking in a healthy and active group of participants, relatively little change in walking parameters was observed until the age of 80 [9]. In this case, it was useful to study participants from a wide age range, rather than to define 'young' and 'old' groups, so that the trends associated with age could be observed. These data also helped the authors to compare the effects of ageing with the effects of early knee osteoarthritis [10].

Extending our previous work on basic walking, the present study aimed to assess walking ability in a controlled environment that simulated some of the features of the built environment; these included stairclimbing, walking on uneven surfaces, walking up and down slopes and on cross-slopes, and getting on and off platforms. We hypothesised that age-related effects would be apparent at an early age in more challenging tasks, compared with those observed in a simple gait assessment on a conventional flat surfaces.

\section{Methods}

\section{Ethics and consent}

The study was approved by the Research Ethics Committee of University College London, UK, and all participants gave their informed consent to participate in the study.

\section{Study population}

Seventy-one participants were recruited in the age range 18 to 92 . All were active, with no self-reported conditions that could affect their ability to complete the course. All were able to complete the course unaided without the need to rest between tasks.

\section{Environment}

The study was performed in the Pedestrian Accessibility and Movement Environment Laboratory (PAMELA) at University College London, UK. PAMELA consists of a modular platform that can be adjusted to simulate different surface types and profiles. It has 36 modules $(1.2 \mathrm{x} 1.2 \mathrm{~m})$ with interchangeable surfaces; many of the modules are divided diagonally. The platform was configured into a series of $9 \mathrm{~m}$ walkways:

1) A standard flat even surface

2) An uneven surface

3) Up and down slopes at an $8 \%$ incline/decline (representing the recommended maximum slope angle for access in European building regulations)

4) A cross-slope at $4 \%$ (common in walkways)

5) Two $1.2 \mathrm{~m}$ long obstacles, $7.5 \mathrm{~cm}$ and $15 \mathrm{~cm}$ high

An illustration of the laboratory set-up in shown in Figure 1. In addition, a flight of 5 steps, intended to access the platform, was also used for measurements. 


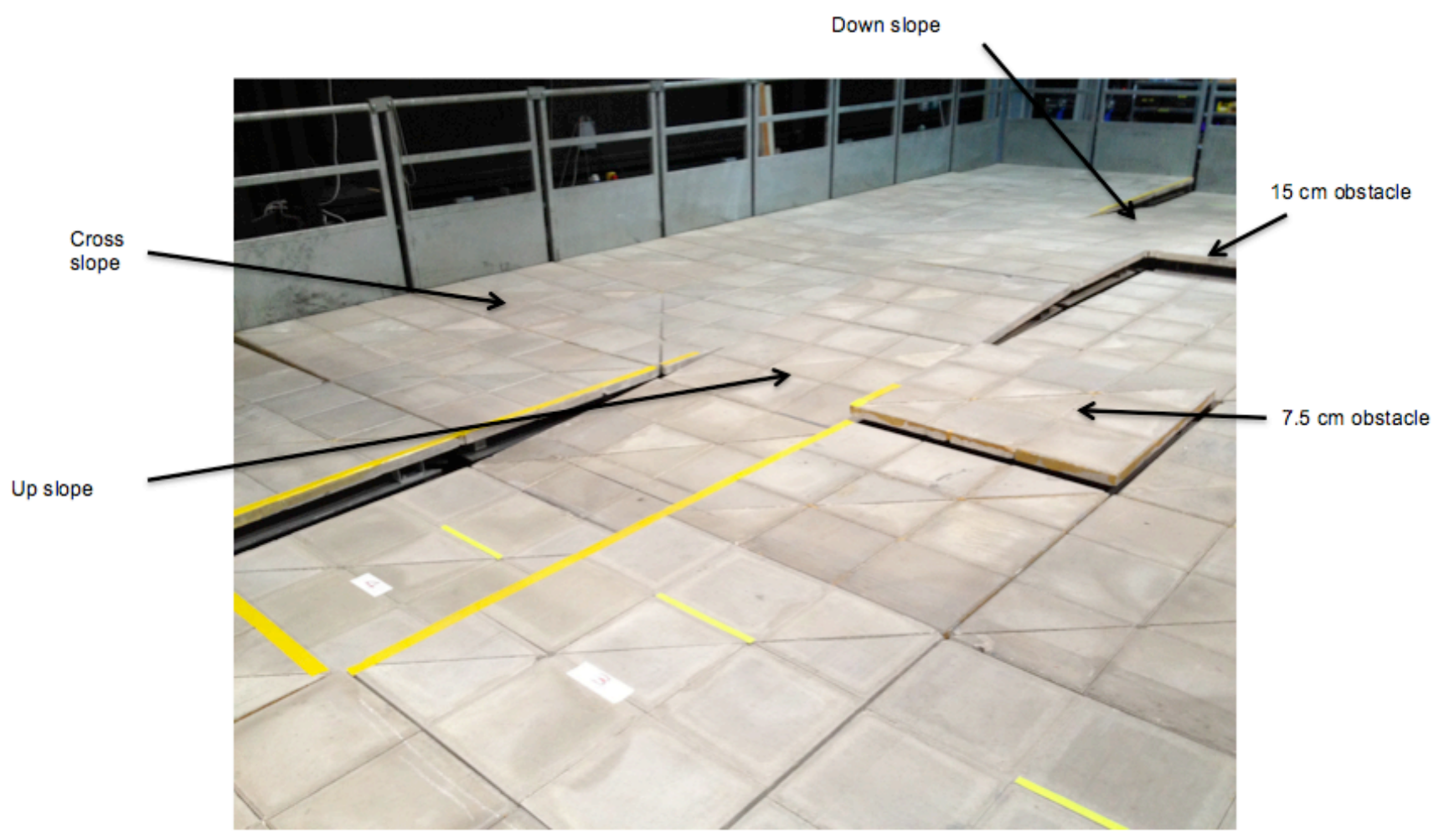

Figure 1. Partial view of the study set-up in PAMELA, illustrating the walkways with obstacles, up and down slopes, and cross slope

Participants were asked to walk in one direction along each walkway, stop and stand still for a few seconds, turn and walk back, before moving on to the next walkway. Walkways were navigated in the following sequence with no rest in between: stairs; flat surface; uneven surface; slope; obstacle; cross slope; flat surface.

\section{Measurements}

Gait was measured using MTw inertial measurement units (IMUs; Xsens Technologies B.V., Enschede, The Netherlands). Five sensors were used in total: one on each thigh and shank, and one attached to the sacrum. The sensors comprise three orthogonal accelerometers, gyroscopes and magnetometers. IMUs on the thigh and shank were attached to rigid plastic bars, which were placed horizontally on the anterior aspect of each limb segment. These were kept in place with tight elastic bandages placed around each segment. Positioning of the IMUs on the shank and thigh were similar to those described previously (Monda et al, 2015). The IMU for the spine was attached to the control unit. At the start of each task, participants were asked to stand still for a few seconds, and an alignment reset was performed. At the end of the measurement procedure, data were exported as .csv files to MATLAB (Mathworks, Natick, USA) for further analysis using custom scripts. The start of each step was defined as the first peak in vertical acceleration after the maximum sagittal shank angle; an example of the vertical acceleration from the shank sensor is shown in Figure 2. For negotiating the obstacles, three steps were considered: i) up, in which the lead foot is on the obstacle and the trailing foot is on the initial level; ii) on, for the first step when both feet are on the obstacle; and iii) down, when the trailing foot is still on the obstacle and the lead foot is down on "ground level". 


\section{Statistical analysis}

Repeated measures ANOVA was used to compare step times for the different activities. Age-related changes of gait parameters were analysed with linear or non-linear regression.

\section{Results}

\section{Participants}

Details of participants' age and gender distribution, height, weight and BMI are summarised in Table 1.

Table 1. Demographic description of the participants in the study

\begin{tabular}{llllll}
\hline & $\begin{array}{l}\text { Age } \\
\text { (years) }\end{array}$ & Male:female & $\begin{array}{l}\text { Height } \\
(\mathrm{m})\end{array}$ & $\begin{array}{l}\text { Weight } \\
(\mathrm{kg})\end{array}$ & $\begin{array}{l}\mathrm{BMI} \\
\left(\mathrm{kg} / \mathrm{m}^{2}\right)\end{array}$ \\
\hline Mean & 48.81 & $39: 32$ & 171.68 & 73.21 & 24.74 \\
SD & 19.25 & & 8.58 & 14.81 & 4.20 \\
\hline
\end{tabular}

\section{Step duration}

Mean values of step duration for all activities are plotted in the graph shown in Figure 3. No significant differences in step duration were observed between walking on an even surface and walking on uneven, cross, upslope or up steps for $7.5 \mathrm{~cm}$ and $15 \mathrm{~cm}$ obstacles. Significant differences were observed in walking down the slope and getting on and off the obstacles. By far the largest differences from level walking occurred when getting on and off the $15 \mathrm{~cm}$ obstacle, and in stair ascent and descent (the four rightmost points in Figure 3); these differences were all statistically significant compared to level walking.

Graphs of age-related changes in step duration are shown in Figure 4 for even, uneven, cross and upslope walking activities. Although no statistically significant differences were found between mean values, it can be seen that step duration increases linearly with age, by about $0.2 \%$ a year. Step duration variability also increased by about $4 \%$ a year for walking on even and uneven surfaces (these were the only two activities that generated enough steps to be able to calculate variability). For more challenging activities (as judged by having a statistically significant difference from level walking), age was shown to have a much greater impact on step duration, as shown in Figure 5. We observed a non-linear relationship with stair climbing, with a third-order polynomial line of best fit (Figure 5a). The age relationship was not so clear for stair descent or getting on and off the $15 \mathrm{~cm}$ obstacle, though fitting the data with a third-order polynomial improves the coefficient of determination compared with a linear plot $\left(0.726\right.$ for a $3^{\text {rd }}$ order polynomial against 0.495 for a linear fit).

\section{Touch down angles}

Touch down angles for the shank, knee and thigh during the various tasks are shown in Table 2. Shank angle was relatively constant across most activities, only varying between $16^{\circ}$ and $21^{\circ}$; it was maintained at a more constant angle than thigh and knee angles. It was only when descending from the $15^{\circ}$ obstacle and for stair ascent and descent that the mean shank angle changed significantly $(\mathrm{p}<0.05)$. Examples of the effect of age on shank angles at touch down are shown in Figure 6 for stair ascent and descent, and for getting on and of the $15 \mathrm{~cm}$ platform. A clear age-related effect was observed for stair ascent, with the shank angle becoming more vertical with age, particularly after the age of 60 years. 


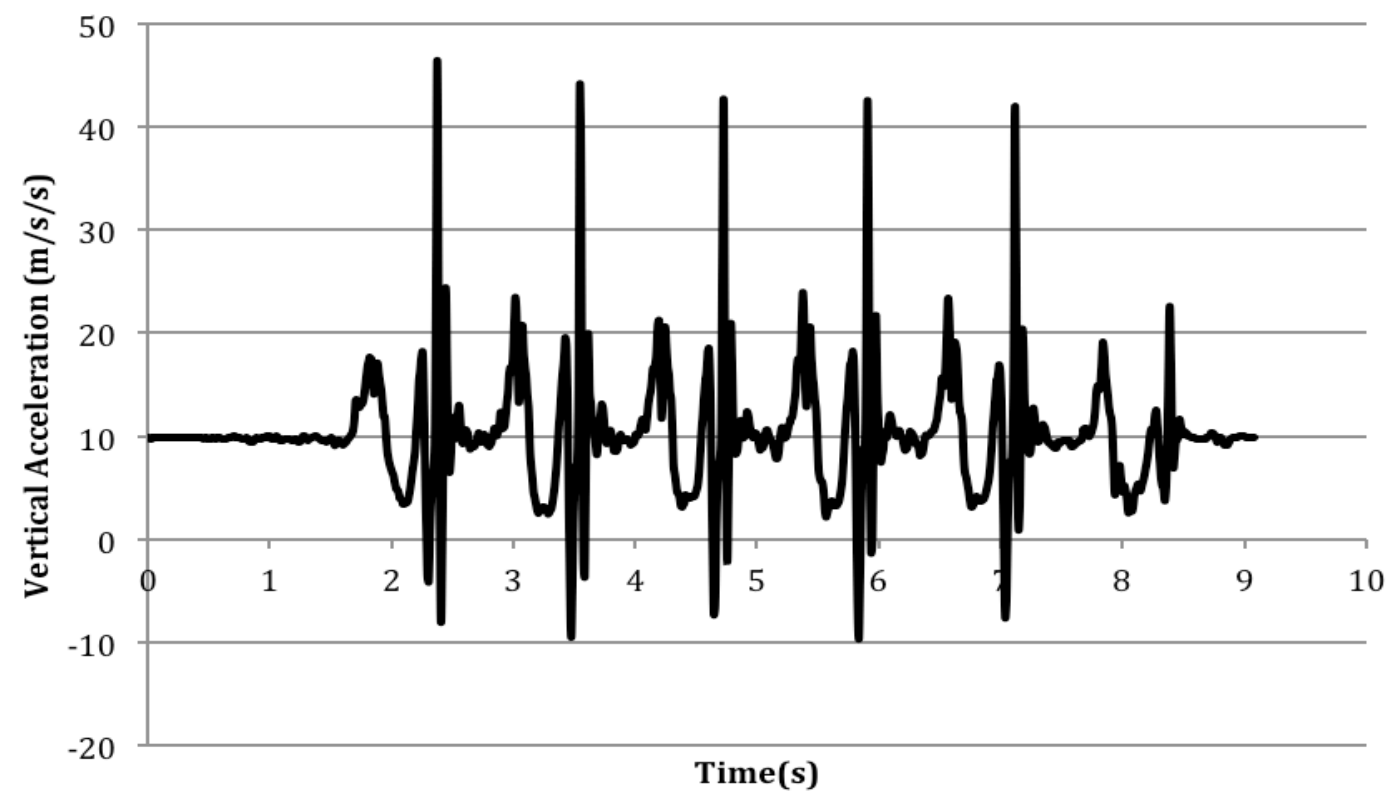

Figure 2. Outputs from shank sensor showing the vertical component of acceleration $\left(\mathrm{m} / \mathrm{s}^{2}\right)$, demonstrating the large acceleration peaks during heel strike enabling the temporal characteristics of gait to be calculated.

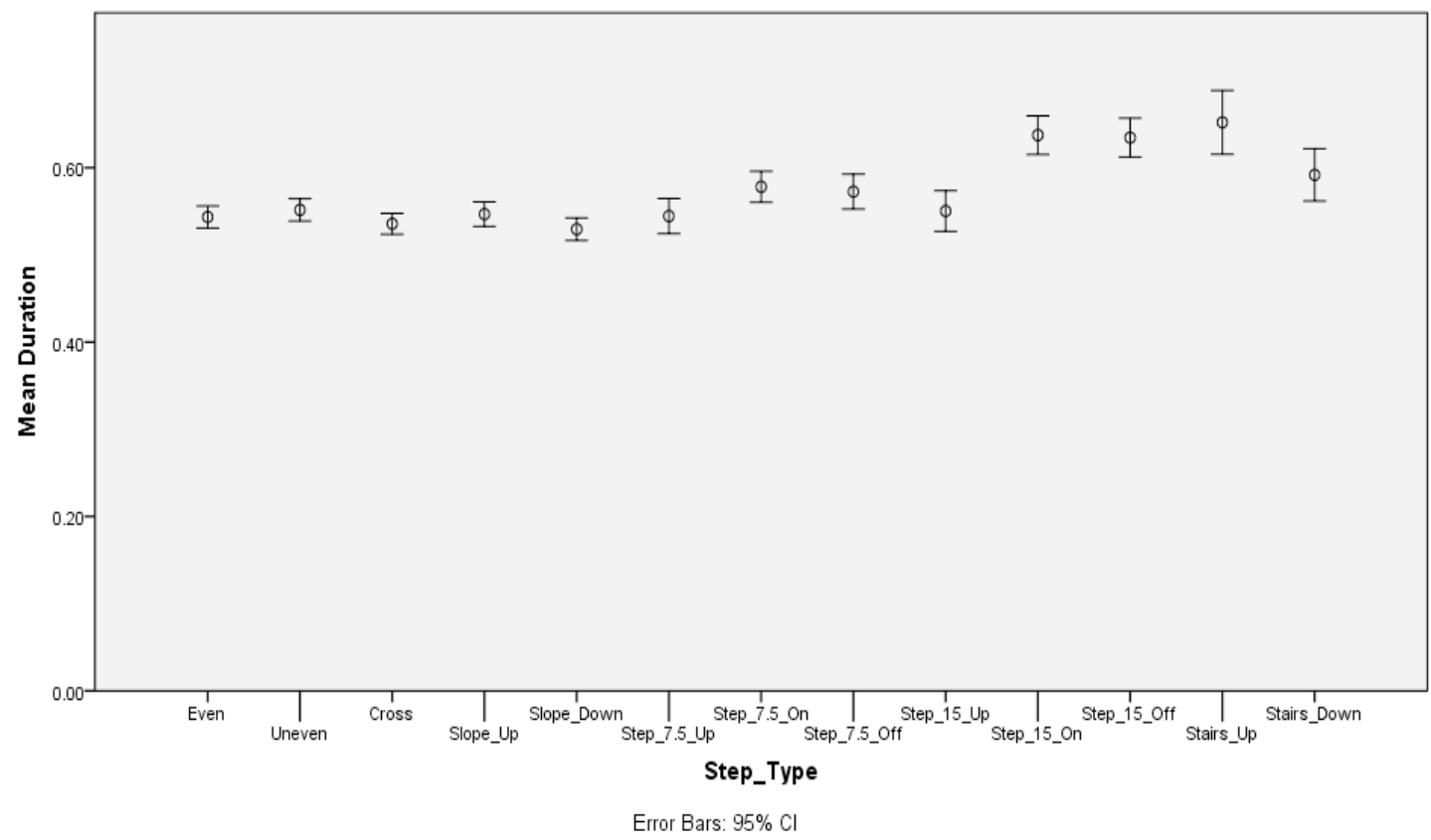

Figure 3. Mean values of step duration 
(a)

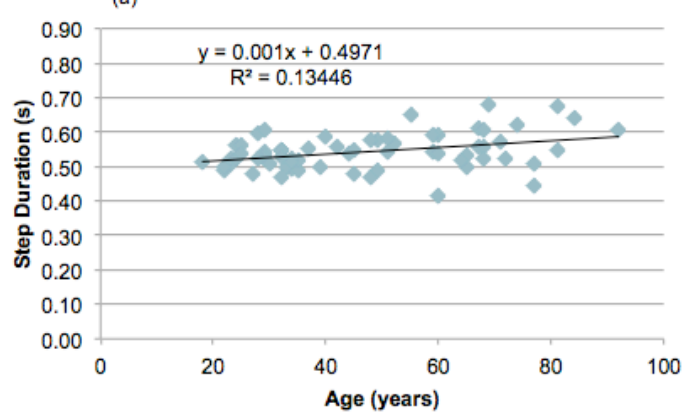

(c)

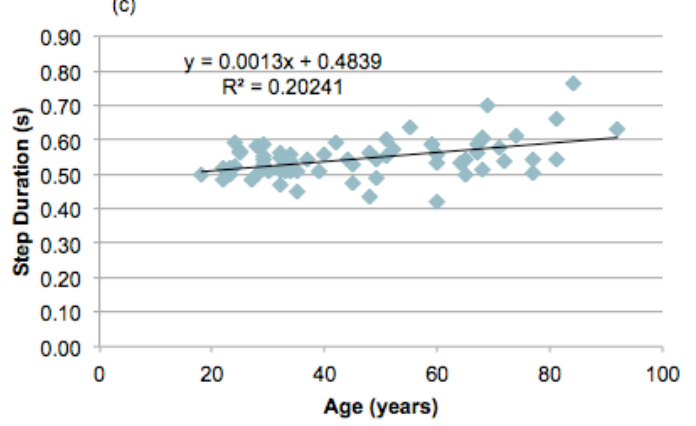

(b)

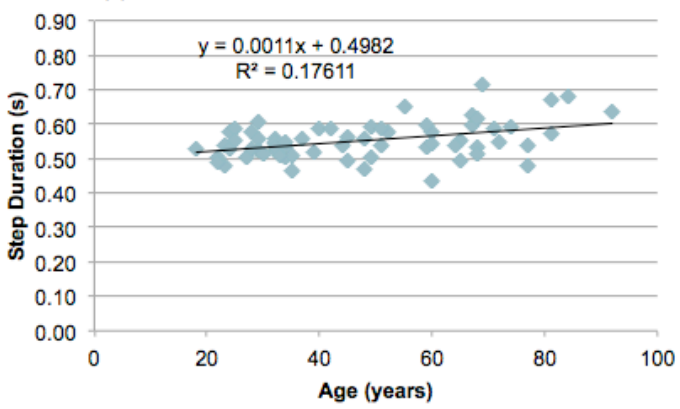

(d)

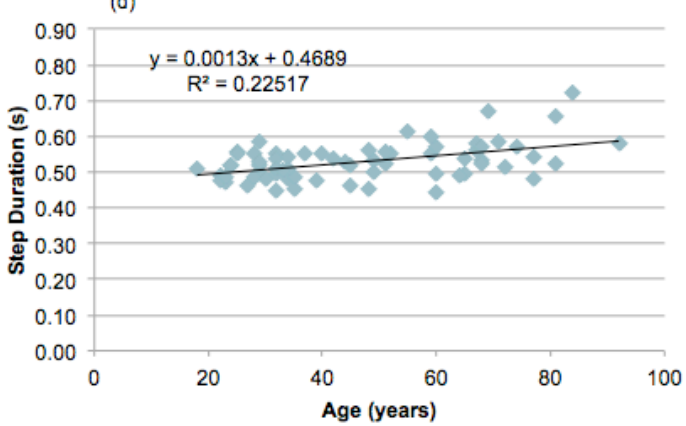

Figure 4. Age-related changes in step duration for walking. Walking on a) an even surface; b) an uneven surface; c) up an $8 \%$ slope; and d) down an $8 \%$ slope. Equations for linear regression of step duration and age are shown on each graph, together with the associated coefficient of determination $\left(\mathrm{R}^{2}\right)$ value.

(a)

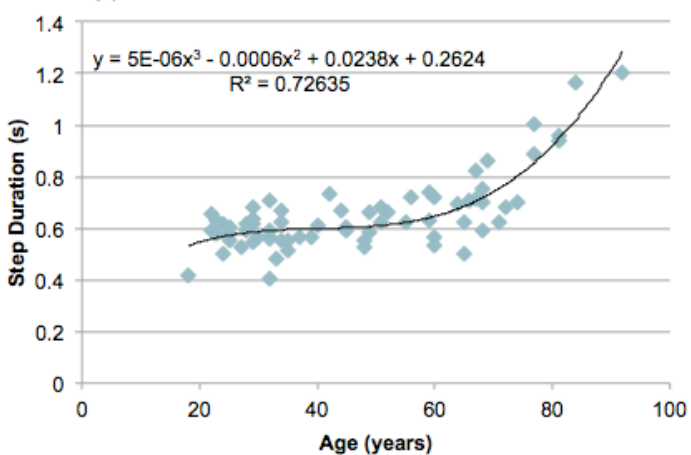

(c)

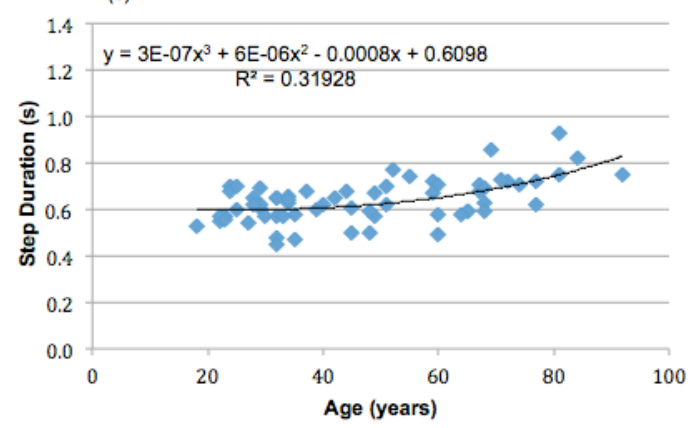

(b)

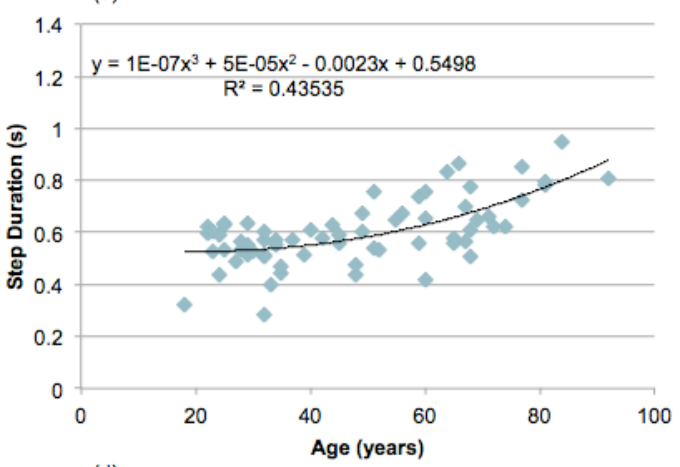

(d)

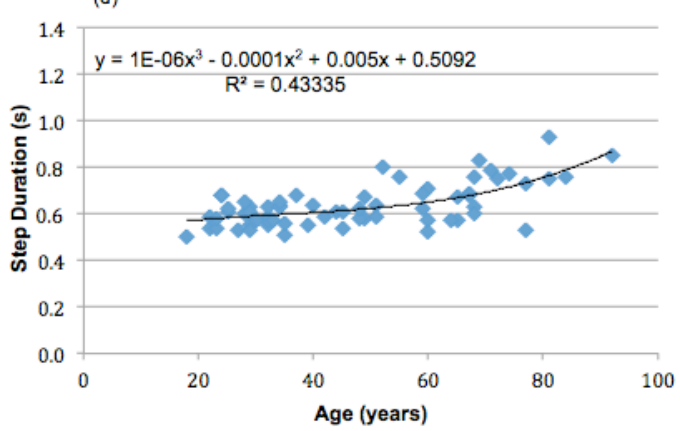

Figure 5. Age-related changes in step duration. Step duration for a) ascending stairs; b) descending stairs; c) getting on to a $15 \mathrm{~cm}$ obstacle; and d) getting down from a $15 \mathrm{~cm}$ obstacle. Equations describing the $3^{\text {rd }}$ order polynomial fit of step duration against age are shown. 
(a)

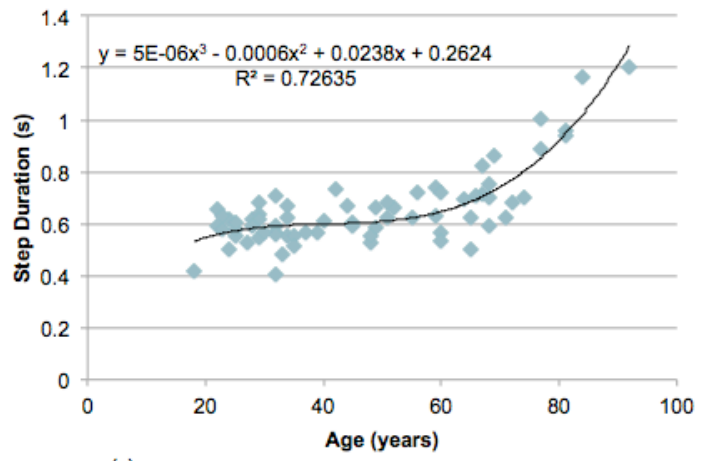

(c)

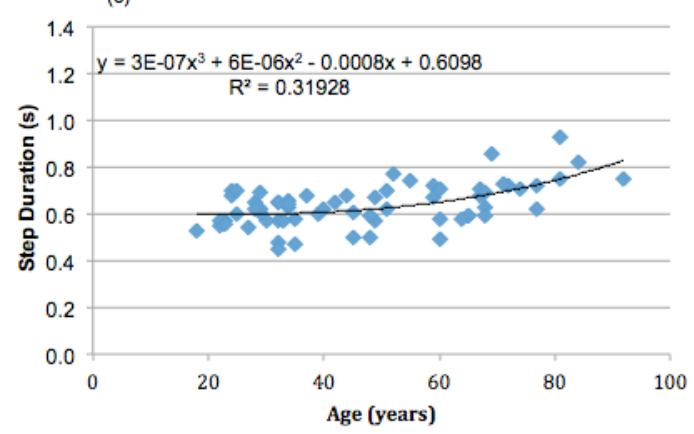

(b)
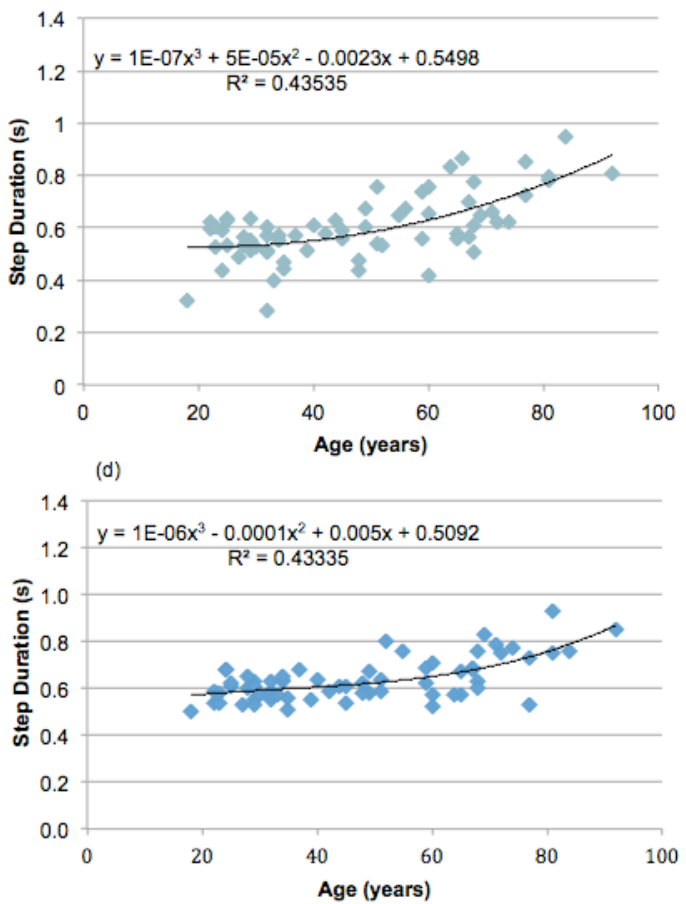

Figure 6. Age-related changes in touch down angle of shank. Touch down angle of shank for a) ascending stairs; b) descending stairs; c) getting on to a $15 \mathrm{~cm}$ obstacle; and d) getting down from a $15 \mathrm{~cm}$ obstacle.

\section{Range of motion}

Range of motion data for the different walking activities are shown in Table 3. Previously, we have shown that these remain relatively constant for level walking in healthy active population until after the age of 80 (Monda et al, 2015).

\section{Discussion}

We recruited participants with a broad range of ages, rather than "young" and "old" groups; this allowed us to better understand how ageing affects mobility in a complex environment. Our participants should be considered as a reference rather than a normal population, because chronic diseases affecting mobility become more prevalent with age, and the healthy older group does not necessarily represent the majority of this population.
The participants in our study found getting on and off the $15 \mathrm{~cm}$ platform and stair ascent to be the most challenging activities with the longest step times. These activities also showed the most significant agerelated effects, with a non-linear increase in step duration over time. It is interesting to consider the advantages and disadvantages of stairs in an older population. Ascending stairs becomes more difficult with increasing age, and therefore poses a greater risk of injury associated with falling down stairs. However, climbing stairs also provides exercise, which can have significant health benefits [4].

Although the height of the $15 \mathrm{~cm}$ obstacle in our study was similar to that of the stair riser, we noted that participants dealt with them in very different ways. Since the obstacle was part of a walkway, getting on to the obstacle was incorporated into the gait action for forward progression; an active contribution of the trailing limb and a passive contribution from inertia both helped participants to raise their centre of mass. 
Table 2. Touch down angles in degrees [mean(s.d)]

\begin{tabular}{llll}
\hline & \multicolumn{1}{c}{ Shank } & \multicolumn{1}{c}{ Thigh } & \multicolumn{1}{c}{ Knee } \\
\hline Even & $21.29(2.89)$ & $27.38(4.64)$ & $4.71(5.19)$ \\
Uneven & $19.74(3.16)$ & $28.13(4.83)$ & $7.59(5.37)$ \\
Cross & $20.56(3.98)$ & $27.76(4.72)$ & $6.12(5.23)$ \\
Slope up & $20.57(4.16)$ & $37.15(5.86)$ & $16.19(6.74)$ \\
Slope down & $15.82(3.27)$ & $24.90(4.94)$ & $8.44(5.91)$ \\
$7.5 \mathrm{~cm}$ up & $16.99(5.77)$ & $37.04(5.63)$ & $20.16(6.97)$ \\
$7.5 \mathrm{~cm}$ on & $16.74(4.95)$ & $26.21(5.16)$ & $8.77(6.79)$ \\
$7.5 \mathrm{~cm}$ down & $13.82(5.57)$ & $24.66(5.59)$ & $10.80(6.72)$ \\
$15 \mathrm{~cm}$ up & $16.13(6.91)$ & $50.51(5.60)$ & $34.62(8.65)$ \\
$15 \mathrm{~cm}$ on & $17.36(5.77)$ & $29.31(6.41)$ & $11.44(7.15)$ \\
$15 \mathrm{~cm}$ down & $5.65(7.84)$ & $21.58(6.17)$ & $15.93(7.43)$ \\
Stairs up & $-17.31(5.27)$ & $42.81(6.53)$ & $60.22(7.63)$ \\
Stairs down & $-2.34(2.51)$ & $14.58(3.11)$ & $16.49(4.74)$ \\
\hline
\end{tabular}

Table 3. Range of motion angles in degrees [mean(s.d)]

\begin{tabular}{lcccc}
\hline & Shank & Thigh & Knee & $\begin{array}{c}\text { Knee } \\
\text { Stance }\end{array}$ \\
\hline Even & $82.6(7.5)$ & $46.1(5.7)$ & $66.2(7.6)$ & $22.5(5.5)$ \\
Uneven & $81.0(9.1)$ & $45.9(6.3)$ & $64.4(9.6)$ & $22.1(6.0)$ \\
Cross & $83.8(7.9)$ & $47.5(6.1)$ & $65.0(8.6)$ & $23.3(5.8)$ \\
Slope 1 & & & & \\
Cross & $82.8(7.6)$ & $47.0(6.1)$ & $64.6(7.8)$ & $23.3(5.0)$ \\
Slope 2 & & & & \\
Slope Up & $81.3(7.8)$ & $54.1(6.2)$ & $65.4(7.7)$ & $30.3(6.1)$ \\
Slope & $78.3(8.9)$ & $38.6(5.9)$ & $70.2(9.8)$ & $26.4(4.9)$ \\
Down & & & & \\
\hline
\end{tabular}

Stair climbing must rely on the muscle power of the leading leg to raise the centre of mass, and does not permit a transfer of kinetic to potential energy, as seen in gait. This seems to be increasingly difficult with age, as demonstrated by the significant increase in step duration for stair ascent for older participants shown in Figure 4a. This may also be related to the increasingly vertical shank angle at touch down observed with increasing age for stair climbing. A more vertical shank will reduce the external flexion moment about the knee during stair ascent, thus reducing the muscle force required. Reduced muscle power may therefore explain the increased time required for stair climbing with ageing. Peak muscle power decreases with age [11]; previous studies have suggested that, in healthy ageing, this does not impact on locomotor function for level walking until after the age of 80 [9]; however, the present study indicates impacts on stair climbing ability begin about 20 years earlier.

Although shank angle at touch down varied with age for stair ascent and descent, it remained relatively constant in other activities, both for comparison between walking on different surfaces and for agerelated effects. The shank angle is towards the end of the kinematic chain in the leg, dependent on both thigh (hip) and knee angle. This indicates a degree of motor control, in that the most distal is the most conserved, rather than the least conserved. It also shows that this motor control can be maintained in older age.

Walking is a very efficient activity; even getting on to relatively high obstacles can be efficient when part of a walking cycle, whereby exchange between kinetic and potential energy is possible. It may not be possible to utilise this exchange in the crowded built environment when forward momentum is impeded, e.g. when getting on a bus or train.

This study of active adults identifies the required capabilities of dealing with complex terrains. However, with ageing, the incidence of mobility limiting chronic diseases affect the ability to deal with the challenges of real world environments, and the results presented in this paper cannot necessarily be extrapolated to the older population in general. Older adults with osteoarthritis, cardiovascular disease or defects of vision are most likely to have very different responses from each other as well as from the healthy active population. In addition, cognitive impairment in older persons can impact on performance [12]. Further research is necessary to understand the separate functional abilities of each group, in order to develop rational strategies to improve mobility and accessibility.

\section{Acknowledgements}

We are grateful to Derek Boampong, Harry Rostron, Xenia Karela and Reka Solymosi for their help in organising the study. 


\section{References}

1. Matthis JS, Fajen BR. Humans exploit the biomechanics of bipedal gait during visually guided walking over complex terrain. Proc Biol Sci. 2013;280(1762):20130700.

2. Lu TW, Chen HL, Chen SC. Comparisons of the lower limb kinematics between young and older adults when crossing obstacles of different heights. Gait Posture. 2006;23(4):471-9.

3. Gaina B, Peters A, Murphy AT, Morris ME. Obstacle crossing deficits in older adults: a systematic review. Gait Posture. 2009;30(3):270-5.

4. Donath L, Faude O, Roth R, Zahner L. Effects of stairclimbing on balance, strength, resting heart rate, and submaximal endurance in healthy seniors. Scand J Med Sci Sports. 2014;24(2):e93-101.

5. McIntosh AS, Beatty KT, Dwan LN, Vickers DR. Gait dynamics on an inclined walkway. J Biomech. 2006;39(1):2491-2502.

6. Marigold DS, Patla AE. Age-related changes in gait for multi-surface terrain. Gait Posture. 2008;27(4):689--96.

7. McCormack GR, Shiel A. In search of causality: a systematic review of the relationship between the built environment and physical activity among adults. Int $\mathbf{J}$ Behav Nutr Phys Act. 2011;8:125.

8. Tyler NA. Capabilities and accessibility: a model for progress. Journal of Accessibility and Design for All. 2011;1(1):11.

9. Monda M, Goldberg A, Smitham P, Thornton M, McCarthy I. Use of inertial measurement units to assess age-related changes in gait kinematics in an active population. J Ageing Phys Act. 2015;23(1):18-23.

10. McCarthy I, Hodgins D, Mor A, Elbaz A, Segal G. Analysis of knee flexion characteristics and how they alter with the onset of knee osteoarthritis: a case control study. BMC Musculoskelet Dis. 2013;14:169.

11. Runge M, Rittweger J, Russo CR, Schiessl H, Falsenberg D. Is muscle power output a key factor in the age-related decline in physical performance? A comparison of muscle cross-section, chair-rising test and jumping power. Clin Physiol Funct Imaging. 2004;24(6):335-40.

12. Persad CC, Jones JL, Ashton-Miller JA, Alexander NB, Giordani B. Executive function and gait in older adults with cognitive impairment. J Gerontol A Biol Sci Med Sci. 2008;63(12):1350-5. 